\title{
Autonomic Dysfunction and Risk Stratification Assessed from Heart Rate Pattern
}

\author{
A. Günther*, O.W. Witte and D. Hoyer \\ Department of Neurology, Friedrich-Schiller-University of Jena, Erlanger Allee 101, D-07747 Jena, Germany
}

\begin{abstract}
The modulation of the autonomic nervous system (ANS) under physiological and pathophysiological conditions is in focus of recent research. Many patients with cardio- and cerebrovascular diseases display features of sympathovagal dysregulation. Measuring specific ANS parameters could improve risk stratification. Thus, the early diagnosis of ANS dysfunction in these patients poses a great challenge with high prognostic relevance.

The most relevant methods and measures of Heart Rate Variability (HRV) analysis and HRV monitoring will be described in detail in this chapter. The grown importance of these easily obtainable heart rate patterns in stratifying the risk of patients with myocardial infarction and heart failure as well as ischemic stroke will be demonstrated based on recent clinical studies. In order to perspectively improve clinical management of these patients further large scale clinical investigations on the role of ANS dysfunction will be useful.
\end{abstract}

Keywords: Autonomic nervous system, heart rate variability, heart failure, stroke.

\section{INTRODUCTION}

Recent research has made important progress in characterizing the role of the autonomic nervous system (ANS) under physiological and pathophysiological conditions as an integrative component in the interorgan interplay.

Early diagnosis of ANS dysfunction within intensive care patients poses a challenge as the majority will go on to display features of sympatho-vagal dysregulation during the course of the disease (e.g. [1]).

Through the measurement of specific ANS parameters clinicians and researchers are enabled to improve cardiovascular and cerebrovascular risk stratification.

The following chapter briefly outlines ANS functions and interactions and summarizes the most relevant methods of its measurement through Heart Rate Variability (HRV) analysis. Prognostic evaluation of critical care patients using such a cost and time-efficient tool as a Holter-ECG in conjunction with clinical scores have been assessed in a number of clinical studies. Here, we will review the present evidence from these studies for such highly relevant pathophysiological conditions of heart failure and myocardial infarction with focus on prognosis of sudden cardiac death, and stroke with a special emphasis neurological outcome related to ANS dysfunction.

\section{THE AUTONOMIC NERVOUS SYSTEM (ANS)}

Autonomic nervous system (ANS) responses to (patho-) physiological stimuli have been characterized by a complex interaction between the different components of the ANS on the one hand, and visceral organs and (involuntary)

*Address correspondence to this author at the Department of Neurology, Friedrich-Schiller-University of Jena, Erlanger Allee 101, D-07747 Jena, Germany; Tel: ++4936419323417; Fax: ++4936419323402;

E-mail: albrecht.guenther@med.uni-jena.de innervated glands on the other hand. In conjunction with the sensory and motor nervous system, the autonomic nervous system (ANS) is responsible for fast, continuous and normally subconscious control of basic physiological functions, such as heart rate, blood pressure, respiratory rate, body temperature and gastrointestinal motility. Forming a functional network of peripheral and central mechanisms throughout the body the autonomic nervous system, however, has two basic divisions: the parasympathetic and the sympathetic component. The classical thinking that parasympathetic and sympathetic nervous system are usually acting in opposition holds true for many ANS functions as in cardiovascular, respiratory as well as gastrointestinal and urinary tract innervations and reactions. But in many other situations the two divisions work synergistically in response to a simultaneous sympathetic and vagus nerve stimulus.

The ANS consists of efferent neurons running in series to innervate smooth musculature of all viscerals and vessels, the heart and glands. Afferent neurons relay visceral input to different parts of the brain. The sympathetic postganglionic transmitter is norepinephrine, whereas the parasympathetic postganglionic transmitter is acetylcholine.

ANS, however, also interacts with different brain structures. The central and peripheral autonomic information is processed by the central nervous system, involving forebrain structures (frontal premotor cortex), hypothalamus, pituitary, amygdala, and the insular cortex. Included in this central network are also several brainstem structures, such as nucleus tractus solitarius (NTS), dorsal motor nucleus of the vagus (DMN), and nucleus ambiguous (NA).

To generate a homeostatic signal level three pathways are relevant: the hypothalamo-pituitary-adrenal (HPA) axis, the parasympathetic and the sympathetic nervous system [2]. Hypothalamic signal input results in the release of corticotrophin-releasing factor from the paraventricular nucleus, by which the HPA axis and the sympathetic nervous system as 
well as the parasympathetic system are modulated. The HPA axis activation finally leads to production of glucocorticoids, resulting in immunomodulation among other hormone related effects.

Peripherally, afferent vagus nerve fibers forward visceral (inflammatory) signals to the brain. Inhibition of inflammation by suppressing pro-inflammatory cytokine release through efferent vagus nerve signals (mediated by acetylcholine, the principle vagal neurotransmitter) has been proposed as the cholinergic anti-inflammatory pathway [3]. Synergistic with the parasympathetic nervous system, the sympathetic activation and secretion of epinephrine and norepinephrine from the adrenal medulla and release from sympathetic nerve terminals also results in beta adrenergic mediated immunological and autonomic modulation $[4,5]$.

These mechanisms aim to keep sympatho-vagal homeostasis. The role of the ANS, however, remains to be further elucidated as a possible key player in the bodies' response to pathophysiological conditions in order to consolidate cardioand cerebrovascular and immune function as well as to develop implications for early diagnosis and treatment of respective dysfunctions.

\section{METHODS OF HRV ANALYSIS}

\section{3.a. Introduction of Linear and Nonlinear Methods}

The fluctuations of heart rate essentially reflect modulations mediated by the autonomic nervous system. They provide insights into different parts of autonomic control as well as risk indices in various pathological entities. In several studies traditional time and frequency domain measures of heart rate variability (HRV) have been investigated in conjunction with cardiovascular outcomes as an important compartment of risk stratification. Furthermore, non-linear HRV measures, such as fractal scaling exponents, complexity indices and indices based on Poincarè plots have been applied in this context.

All of these measures are based on electrocardiographic (ECG) recordings. By means of mobile so called "Holter ECG" systems, the patient's HRV can be assessed under "normal" daily activity. In clinical settings the ECG can be recorded both by means of Holter systems and by stationary bedside monitoring systems. In order to obtain a reasonable precision of the heart rate fluctuations the ECG should be sampled with rate of at least $1000 \mathrm{~Hz}$. The heart beats have to be exactly identified for example by template matching or a steepest ascent criterion followed by an annotation of normal and arrhythmic beats. In order to focus on the autonomic rhythms and discriminate from those beats reflecting cardiogenic arrhythmias the convention of analyzing only intervals between normal heart beats (so called NN intervals) was set more than a decade ago [6]. Clean NN-interval series can be constituted after treating arrhythmias by appropriate interpolation or deletion. The relative number of such removed arrhythmic beats is an important measure of the data quality and can itself provide additional prognostic value beyond the "Task Force HRV" analysis [6].

\section{Indices of HRV Under Spontaneous Conditions}

HRV indices can be calculated from the overall 24 hour recording or from shorter periods which reflect particular situations. Also the circadian rhythm of autonomic activity should be taken into consideration.

Linear HRV assessment of those type of data was first organized by a Task Force of the European Society of Cardiology and the North American Society of Pacing and Electrophysiology in 1996 [6], and has recently been successfully validated in several clinical studies as demonstrated in the studies referred below. These guidelines are still the established HRV standard, even though an update has been recommended $[1,7]$. This mainly "linear" HRV analysis is outlined in more detail below (see 3b).

Nonlinear HRV assessment was proposed by using different mathematical approaches:

Fractal approaches such as the beta-slope in the log-log power spectrum and indices of detrended fluctuation analysis (DFA, [8, 9] assess the self-similar qualities of the HR time series between different time scales. Their success as predictors of outcome supports the potential relevance of measuring complex cardiovascular coordination over a wide range of scales and frequencies, respectively. Normal behaviour is associated with fractal-like correlated properties. Pathophysiological conditions can change the fractal scaling exponent or even distort the self-similarity in favour of single scale rhythms at one extreme or random noise at the other.

Complexity approaches assess the randomness (unpredictability) of the NN interval series. This can be done based on the original data leading to approximate entropy [10], sample entropy ([11], conditional entropy [12], or information loss [13-15]

The accentuation of key patterns by transformation of the $\mathrm{NN}$ interval sequences into sequences of a few symbols leads to complexity measures of symbolic dynamics [16,17].

Those approaches are furthermore a fundamental way of analyzing the information flow that characterizes communication. Cardiovascular control operates by communication between different parts of the autonomic nervous system at different time scales $[13,15]$ Complexity measures as mentioned above are calculated using one selected time scale [10,18] Costa et al. [19] introduced "Multiscale entropy", a complexity function over multiple scales, and demonstrated the relevance of heart rate complexity pattern on different time scales.

Autonomic Information Flow (AIF) functions reflect the information flow over different prediction time horizons and time scales, respectively, of NN interval series. AIF functions allow the assessment of complexity of both short and long term heart rate patterns at scales analogous to those of traditional HRV measures [20]. In that way the complex rhythms and interactions, essentially mediated by the autonomic nervous system were introduced into cardiovascular risk assessment. The capability of AIF analysis opens a new window into the development of physiologically relevant complexity measures by considering underlying autonomic mechanisms and the extensive knowledge base already gained using the standard HRV approaches of task force monitoring [21].

These methods focus on autonomic rhythms, reflected by normal-to-normal $(\mathrm{NN})$ beat intervals after removing and interpolating cardiogenic arrhythmic parts from the meas- 
ured heart beat interval series. On the other hand, cardiogenic arrhythmias such as ectopic beats are of particular diagnostic and prognostic value using different mathematical tools.

Heart Rate Turbulence (HRT) is an alternative method where the heart rate response to premature ventricular complexes (VPC) is evaluated [22]. This response reflects autonomic blood pressure regulation associated activity induced by a decreased heart ejection volume period. Reduced HRT is significantly associated with mortality after myocardial infarction [23].

\section{Heart Rate Responses Under Test Conditions}

Orthostatic tilt test, baroreflex sensitivity by phenylephrine administration, chemoreflex sensitivity by breathing air with increased or decreased oxygen, cardiovascular effects of other drugs, and controlled ventilation are prominent examples of selectively challenging the autonomic system and consequently stimulation of well defined heart rate responses [24,25]. Those tests address particular control loops more specifically than the indices of HRV under resting conditions. Otherwise they are laborious and do not allow a long time monitoring.

The spontaneous and instantaneous heart rate response to blood pressure fluctuations is an index of "spontaneous baroreflex sensitivity". The so-called "alpha-index" reflects the coupling within particular frequency bands of heart rate and blood pressure rhythms [26,27]. The "sequence technique" reflects the coupling between sequences of increasing or decreasing, respectively, heart rate and blood pressure values $[26,28]$.

\section{3.b. "Task Force" HRV Monitoring}

Besides the heart rate fluctuations assessed as HRV measures already the mean heart rate provides valuable information about the autonomic control. The main time and frequency domain HRV measures according to [6] will be introduced as followed.

\section{Selected Prominent Measures of Linear HRV Analysis}

In the time domain different measures of the dispersion of the NN intervals are assessed from the entire data set:

SDNN: Standard deviation of all normal to normal (NN) intervals. This measure reflects the overall variability whereby the slower rhythms contribute stronger due to their larger amplitudes.

RMSSD: Square root of the mean squared differences of successive NN intervals. This measure reflects faster rhythms which are usually assigned to vagal rhythms.

Further related indices are:

SDANN: Standard deviation of the average of NN intervals in all 5 min segments of the entire recording.

SDNNindex: Mean of the standard deviations of all NN intervals for all 5 min segments.

HRVtriangular index: Total Number of all NN intervals divided by the height of the histogram of all $\mathrm{NN}$ intervals.

TINN: Baseline width of the maximum square difference triangular interpolation of the highest peak of the histogram of all NN intervals.
pNN50: Number of pairs of adjacent NN intervals differing by more than $50 \mathrm{~ms}$ divided by the total number of NN intervals in the entire recording.

In the frequency domain the power spectral density reflects the distribution of independent sine waves. Usually the NN interval series is equidistantly re-sampled and power spectra are calculated from the resulting time series. This can be done either by a Fast Fourier Transform algorithm using appropriate windowing or by statistically reliable autoregressive models. Different components of the power spectrum (frequency band power) are assigned corresponding to typical rhythms of the autonomic modulations of the heart period:

The short term rhythms are accentuated by using the mean of the measures obtained by analyzing short windows (5min: HF, 5min: LF, 30min: VLF) in which the entire data set is subdivided:

TP (Total Power): lowest signal frequency- $0.4 \mathrm{~Hz}$ (overall HRV).

VLF (Very Low Frequency): $<0.04 \mathrm{~Hz}$, reflecting the integrative effect of various controllers such as from vagal up to humoral effects [6,29].

LF (Low Frequency): 0.04-0.15 Hz, reflecting vagal and sympathetical mediated control.

LFnorm: LF/(TP-VLF): emphasizes the relative contribution of LF.

HF (High Frequency): 0.15-0.4 Hz, predominantly reflecting vagal mediated control.

HFnorm: HF/(TP-VLF): emphasizes the relative contribution of HF.

LF/HF: ratio interpreted as sympatho-vagal balance.

In Fig. (1) the change of a short term power spectrum due to tilt load is shown. The shift of the sympatho-vagal balance is reflected in the relative increase of LF and decrease of HF band power.

\section{Short-Term Power Spectral Density}

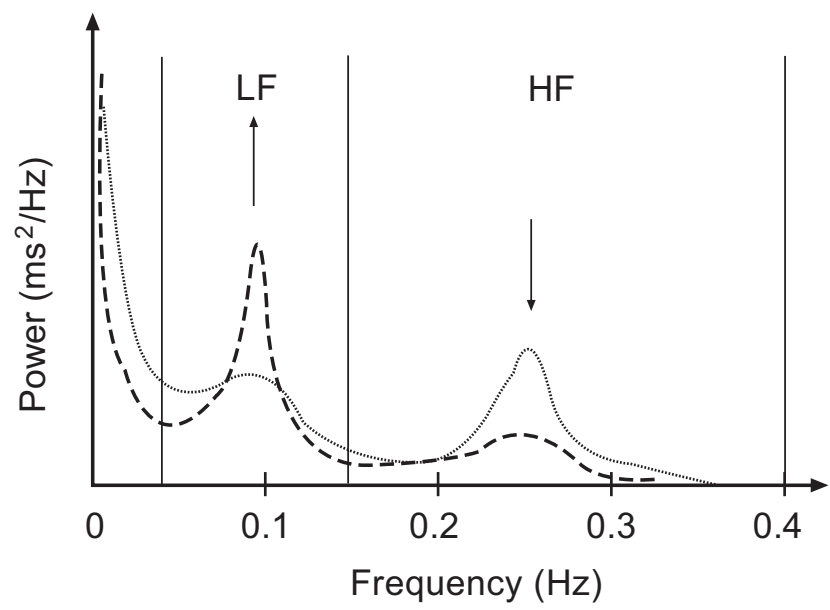

Fig. (1). Sketch of short term HRV frequency domain characteristics according to [6]: Power spectral density during rest (dotted line) and tilt load (dashed line). Note the shift of the sympathovagal balance represented by the LF and HF peaks. 
The long term rhythms are analyzed from the entire data set as a whole. Besides frequency bands corresponding to the short term measures the Ultra Low Frequency (ULF) band is additionally considered. The resulting 24 hours power spectrum mainly reflects the circadian rhythm. The frequency bands of these long term power spectra were found to be predominant predictive. The square root of the total power (TP) is equivalent to SDNN of the same data set. Moreover, the decay of a double logarithmic plot of this power spectrum (beta-slope), which quantifies its fractal behaviour, turned out as important measure of complex multi-scale cardiovascular behaviour.

Beta slope: Slope of a line fitted to the log-log-power spectrum between $10^{-4}$ and $10^{-2} \mathrm{~Hz}$ (Fig. 2).

\section{Long-Term Power Spectral Density}

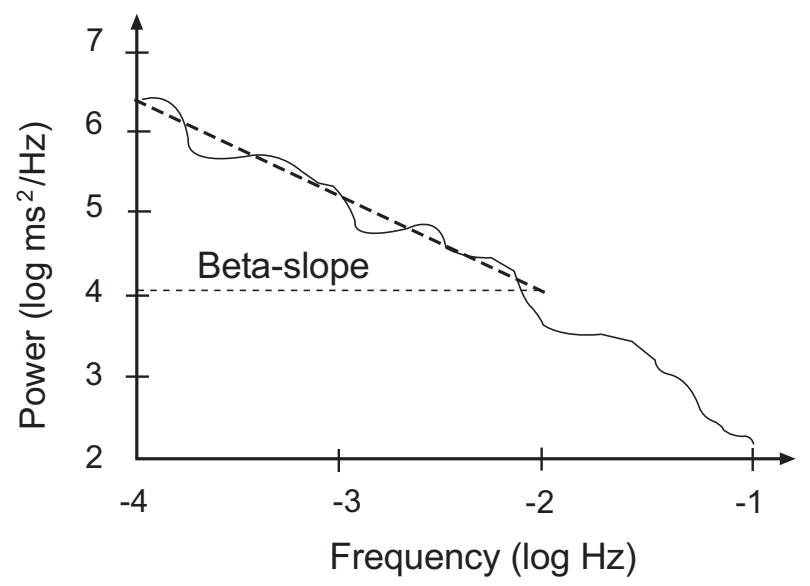

Fig. (2). Sketch of a long term power spectrum. Note the slope of the fitted line between $10^{-4}$ and $10^{-2} \mathrm{~Hz}$, respectively -4 and -2 in the logarithmic scale.

Each of the indices in time and frequency domain has different advantages, and robustness when applied to measured clinical data. Hence the most appropriate of redundant indices (correlations see Table 1) have to be chosen.

The classical physiological interpretation of the mediators underlying the heart rate fluctuations reflects consensus on a simplified level, but also taking into account complex inter-relationships between multiple autonomic, humoral and other processes. Under particular pathological conditions these relationships can even be changed [31]. As a basic rule of dynamic processes can be applied that faster processes influence slower ones, such as discussed for vagal influences reflected in VLF band [29]). In critical care risk stratification parasympathetic indices (RMSSD and $\mathrm{HF}_{\text {short term }}$ ) as well as $\mathrm{VLF}_{\text {short term }}$ provided prognostic value. Some respective implications such as those related to autonomic regulations of inflammation, the activity of the renin-angiotensin system, or respiratory dysfunctions are discussed in [1,32]. Furthermore, reduced SDNN which reflects the overall HRV and hence the effect of multiple different physiological controllers and influences up to the circadian rhythm turned out as risk predictor in several studies, a result that confirms the comprehensive systemic approach of analyzing autonomic rhythms by HRV analysis. The same holds true for Betaslope as a comprehensive long term HRV measure.

The general procedure of (linear) HRV analysis is outlined in Fig. (3) according to [6]. The later developed (nonlinear) complexity and AIF indices are not yet organized following a respective standard scheme. Nevertheless, they are usually assessed following related considerations, however, with focus at complex interactions within autonomic control.

Even though easily applicable, the method of HRV analysis absolutely relies on good signal quality and its major limitation is the preclusion of atrial fibrillation, sinoatrial dysfunction and $>20 \%$ ectopic complexes.

\section{HEART FAILURE AND MYOCARDIAL INFARC- TION}

Myocardial infarction (MI) and heart failure are the leading causes of death and morbidity in industrial countries. Investigations of patients after MI revealed ANS dysfunction as a predictor for cardiac mortality in association with increased incidence of sudden cardiac death [33]. For the general clinical use HRV measures are of particular interest in cases where they add prognostic value independent of the established clinical variables or where they can substitute complicated clinical scores by easily obtainable HRV indices during monitoring.

Therefore, since more than one decade HRV analysis is established in clinical cardiology and accepted as a part of risk assessment concerning sudden cardiac death. Currently, the development of HRV based ICD (implantable cardioverter defibrillator) control parameters is one of the predominant questions.

The autonomic rhythms represented by heart rate patterns reflect certain pathophysiological aspects of several cardiac disorders, like myocardial infarction, congestive heart failure, cardiomyopathy, or ischemic heart disease. The comorbidity of cardiac diseases with for example psychiatric or mental disorders leads to even more complicated identifica-

Table 1. Fundamental Correlations Between Time Domain Measures (Grouped Left) and Frequency Domain Measures (from $[6,30])$

\begin{tabular}{|l|l|l|l|l|}
\hline Correlations & \multicolumn{5}{l|}{ Frequency Domain } & LF & HF \\
\cline { 2 - 5 } Time Domain & TP & VLF & & \\
\hline \hline SDNN, SDANN, HRVtri.ind., TINN & $\mathrm{x}$ & & $\mathrm{x}$ & \\
\hline SDNNindex & & $\mathrm{X}$ & & $\mathrm{X}$ \\
\hline RMSSD, pNN50 & & & & \\
\hline
\end{tabular}




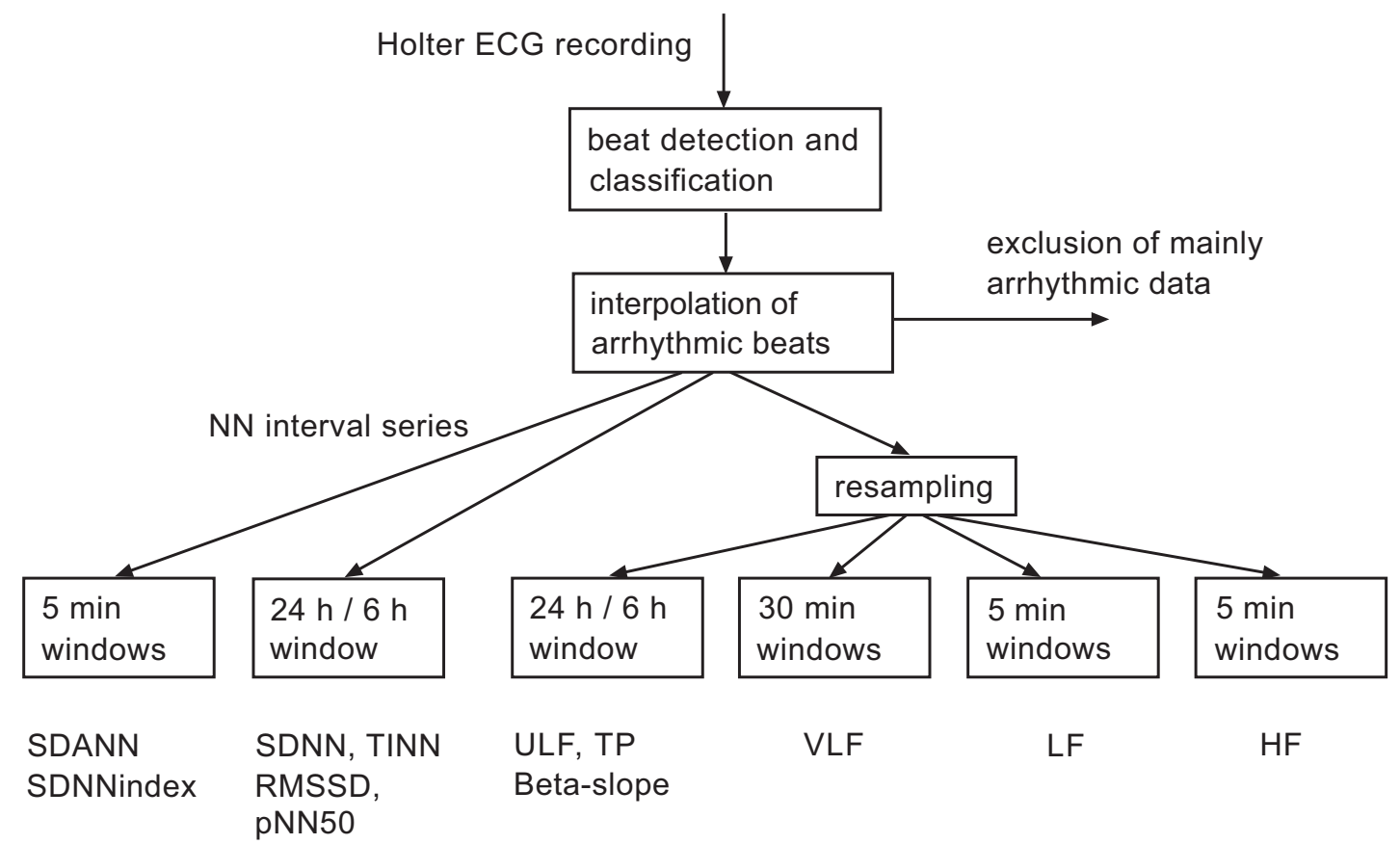

Fig. (3). Flow chart of the established standard HRV analysis according to [6]. Data sets with more than 5\% arrhythmic beats excluded from the analysis. This scheme holds also true for novel complexity and AIF indices.

tion of the origin of a measured HRV pattern. For example, the very low frequency (VLF) band power in depressive in comparison to non-depressive patients (both groups with recent acute myocardial infarction $(\mathrm{MI})$ ) is reduced due to depression and MI, whereby both disorders constitute independent factors of mortality [34].

Additionally, HRV is influenced by current physical activity, sleep states, circadian rhythm, as well as on age of the patient (e.g. [35-42]).

Representative results of larger clinical prognosis studies of patients with cardiac diseases using 24 hours HRV are summarized in Table 2. A comprehensive review of HRV based predictors of mortality after myocardial infarction by Kleiger et al. [24] includes the selected key results presented here. The strongest clinical risk predictor is a reduced left ventricular ejection fraction (LVEF). A main objective of clinical studies is to evaluate whether HRV indices provide independent additional prognostic value.

Reflecting the briefly summarized results presented in Table 2 obviously the reduced overall variability (SDNN, HRV index) or slow rhythms (VLF band power) provide consistent HRV based risk predictors.

In chronic heart failure patients and those with unstable angina also reduced LF or LF/HF showed predictive value. A reduced DFA index (reduced fractal correlations) was found of superior predictive value than SDNN in a study of post-myocardial infarction patients [43].

Due to the interindividual heterogeneity and the complex contributions such as autonomic, mental, psychiatric, physical activity, circadian rhythm, etc. besides the cardiac dysfunction, and furthermore the often unclear causality and comorbidity, it is unlikely to find the "optimal" HRV index. However, selected indices may be sensitive to particular as- pects of pathophysiological autonomic control and hence particular patient groups.

Maestri et al. [44] compared 20 nonlinear indices and SDNN as the best discriminating linear HRV index in a group of 200 patients with chronic heart failure (CHF). They found two nonlinear indices (one from empirical mode decomposition, one from symbolic dynamics) as significant predictors independent of the clinical parameters and SDNN. A third respective set of significant nonlinear predictors of this CHF patient group was found by autonomic information flow (AIF) characteristics [45]. These provided additional predictive value to the best clinical indices NYHA class and LVEF. The Kaplan Mayer survival curves (Fig. 4) show their discriminatory power. The result of this patient group indicates the predictive value of the complex overall autonomic adjustment as superior compared to particular rhythms, such as HF or LF, which did not significantly contribute in that study.

In a scientific statement from the American Heart Association Council on Clinical Cardiology Committee on Electrocardiography and Arrhythmias and Council on Epidemiology and Prevention noninvasive risk stratification techniques for identifying patients at risk for Sudden Cardiac Death (SCD) were reported [46]. LVEF, QRS duration, QT interval, QT dispersion, signal-averaged electrocardiogram, as well as, short-term HRV were concluded as with limited predictive value. Long-term ECG recordings provide information about both, ventricular arrhythmias and long-term HRV. Ventricular arrhythmias were found as an abundant and recommended SCD predictor in post-MI patients. Depressed HRV was confirmed to be a clear predictor of overall mortality in several studies such as also reported in Table 2. In particular in non-arrhythmic mortality long-term HRV may be a better predictor. Finally, HRT, which assesses the autonomic response modulating the heart rate, turned out as 
Table 2. Studies of Heart Rate Related ANS Dysfunction in Heart Failure and Myocardial Infarction Patients

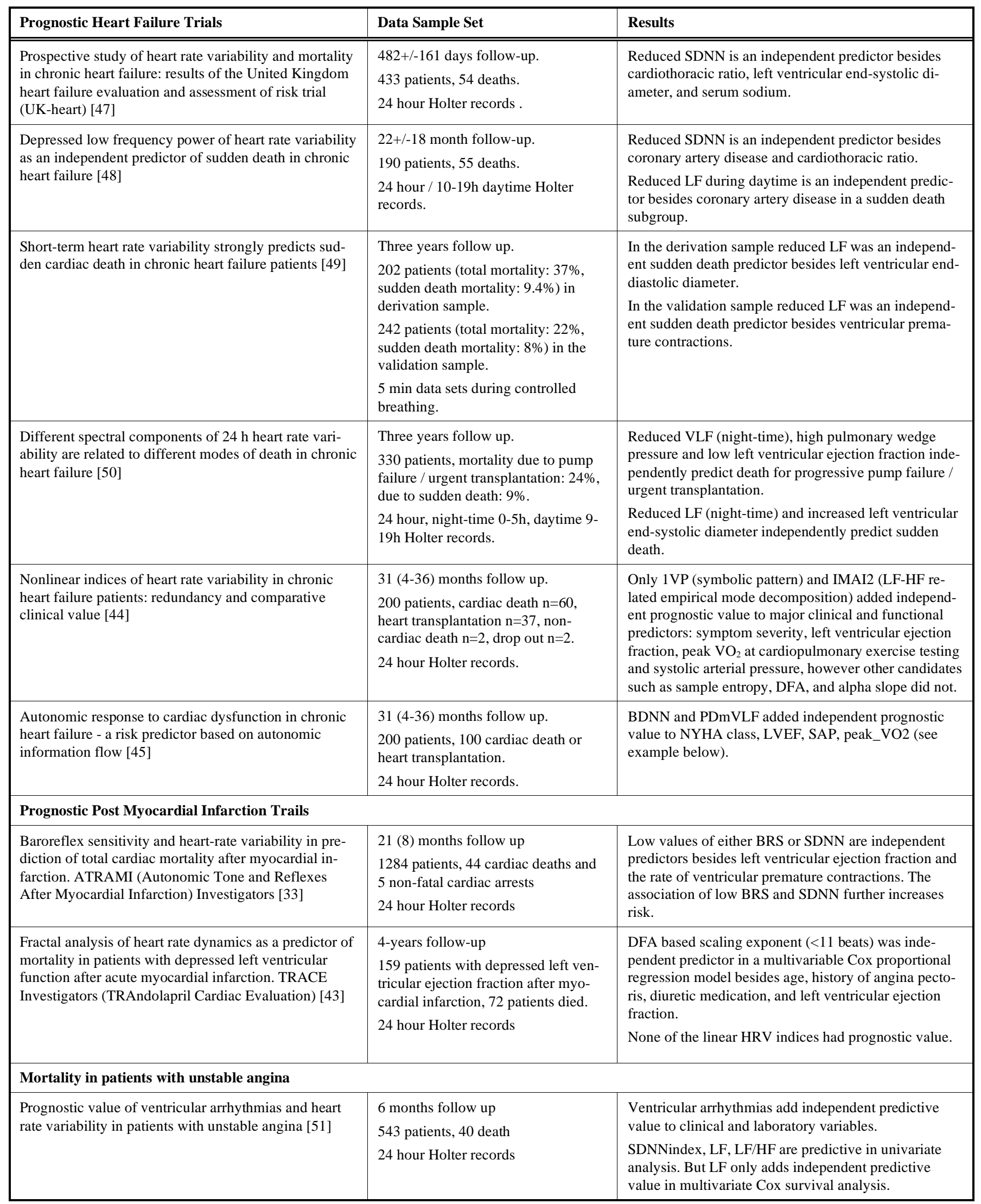

promising candidate for SCD prediction. The latter two results, namely long-term HRV and HRT, confirm the rele- vance of considering the autonomic control and dysfunction, respectively, as independent predictors of SCD. 


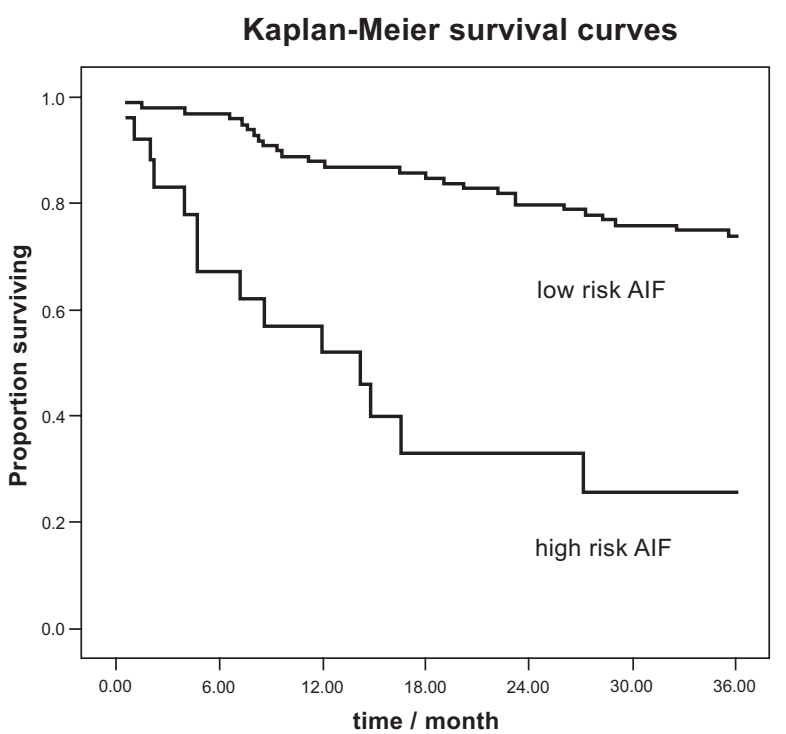

Fig. (4). Kaplan-Meier survival curves for total cardiac mortality, AIF index according to [45].

In conclusion, the key results of the studies summarized in Table $\mathbf{2}$ clearly show the independent prognostic value of the autonomic nervous system related indices in extension to clinical and functional variables. Nevertheless, other influences such as age, gender, history, NYHA class, treatment (thrombolysis, beta-blocker, ACE inhibitors), major depression, etc. were partly considered as confounders of HRV indices.

The consistent prognostic value of long term variability and correlations (SDNN, SDNNindex, VLF, beta-slope) may reflect the importance of the complex adjustment of several underlying mechanisms such as vagal and sympathetic rhythms (Table 2). Those results confirm the importance of complex autonomic adjustments and thus strongly motivate applying AIF analysis in the risk assessment in CHF and MI patients [45]. Furthermore, these results support the importance of a complex systemic analysis, reflecting both the particular cardiac disorder and compensation/adaptation by autonomic and other systemic mechanisms. In conclusion, the established linear HRV analysis provides sensitive markers of the cardiovascular-systemic response to cardiac dysfunctions, which can significantly be extended by novel nonlinear methods.

\section{STROKE}

In stroke as the third most important cause of death in industrial countries a variety of ANS disturbances can occur. They are attributed to the damage of the different compart-

\section{Stroke}

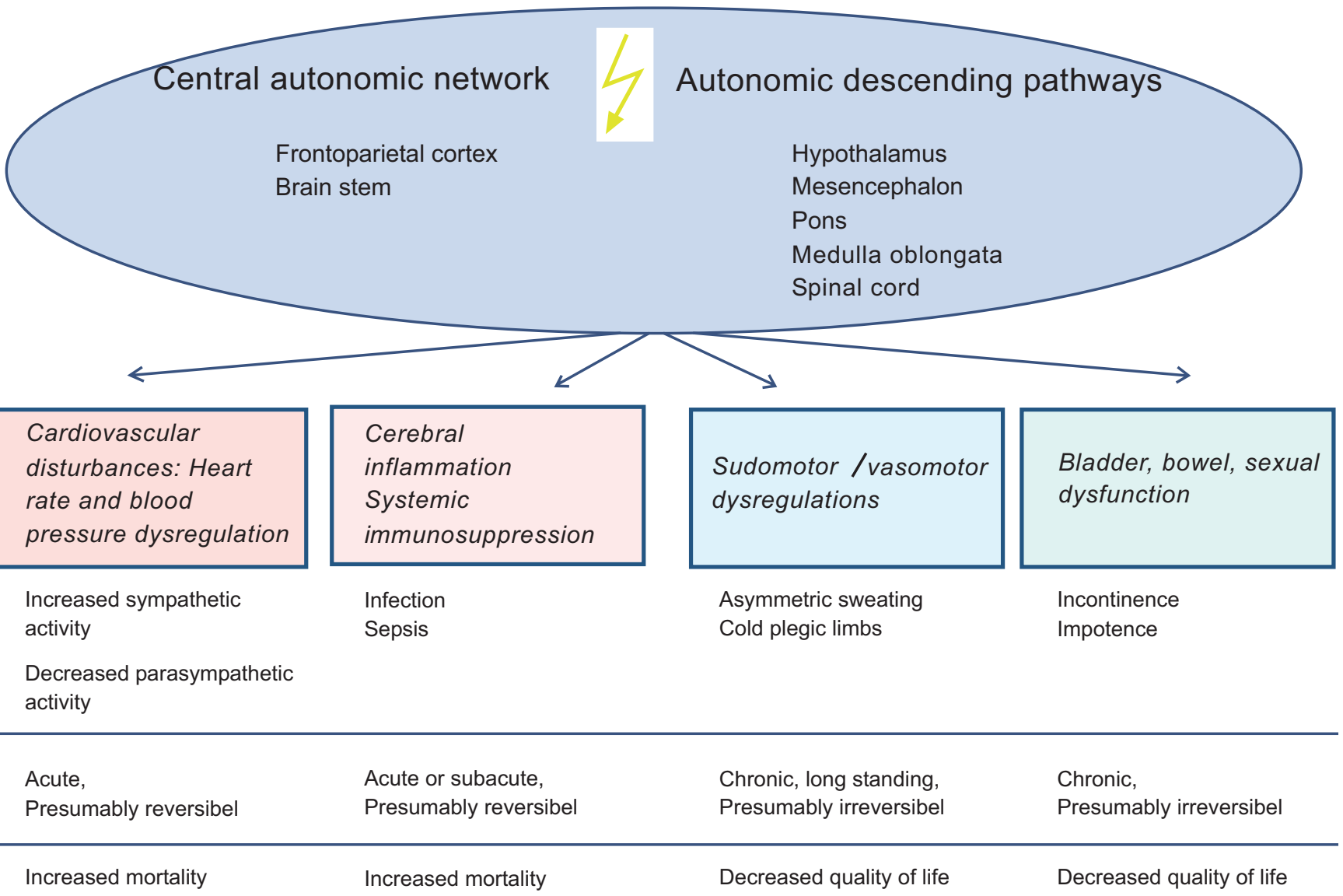

Fig. (5). Stroke related autonomic disturbances and their clinical relevance. 
ments of the central autonomic network, i.e. frontoparietal cortical areas and brain stem (see above, and Fig. 5) or may result from disrupted autonomic pathways (hypothalamusmesencephalon-pons-medulla oblongata-spinal cord). Among the ANS dysfunctions in stroke victims cardiovascular sympathetic/ parasympathetic dysbalance is most evident in the acute phase of stroke. This cardiovascular as well as infection related morbidity within the first days after stroke is very relevant for survival and neurological outcome after stroke [52].

Since the early $1990^{\text {th }}$ studies in ischemic brain infarction revealed frequent ANS dysfunctions in association with pathological activation of the sympathetic system. Such a sympathetic hyperactivation and autonomic imbalance in acute ischemic stroke patients resulted in increased risk and incidence of severe cardiac arrhythmias and sudden cardiac death [53-56].

Based on NN interval analysis Colivicchi et al. showed in 208 stroke patients the best predictive value for 1 year survival using SDNN, however the occurrence of ventricular tachycardia had an even higher predictive value [57]. Interestingly, stroke localization seems to play an important role in this context: HRV dysregulation (i.e. decreased LF, HF and SDNN) was more frequent in association with right hemispheric and insular stroke independent of the use of either time frame or spectral analysis. Right hemispheric brain infarction with insular involvement is associated with the highest degree of ANS dysfunction (measured as de-

Table 3. Clinical Studies of Heart Rate Related ANS Dysfunction in Stroke Patients

\begin{tabular}{|c|c|c|}
\hline Author & Data Sample Set & Results \\
\hline Barron et al., 1994 [53] & $\begin{array}{l}40 \text { stroke patients } 4-11 \text { days after event } v s .40 \text { matched } \\
\text { controls, power spectrum analysis of HRV, all pts. in sinus } \\
\text { rhythm. }\end{array}$ & $\begin{array}{l}\text { Cardiac parasympathetic innervations reduced after } \\
\text { stroke; more pronounced after right sided infarct. }\end{array}$ \\
\hline Naver et al. 1996 [54] & $\begin{array}{l}23 \text { stroke / } 11 \text { TIA patients vs. } 21 \text { matched controls, no } \\
\text { medication except aspirin, follow up } 8-48 \text { days (stroke) up } \\
\text { to } 2 \text { years (TIA), respiratory HRV, orthostatic test, isomet- } \\
\text { ric handgrip. }\end{array}$ & $\begin{array}{l}\text { Right sided stroke associated with reduced respiratory } \\
\text { HRV, peripheral reflexes equal between right an left } \\
\text { lesions. }\end{array}$ \\
\hline $\begin{array}{l}\text { Korpelainen et al. } 1997 \\
{[61]}\end{array}$ & $\begin{array}{l}32 \text { stroke patients } v s .32 \text { matched controls; } 24 \text { h Holter, } \\
\text { time and frequency domain acute and after } 6 \text { months. }\end{array}$ & $\begin{array}{l}\text { LF/HF ratio unchanged during night-time in stroke pa- } \\
\text { tients - reversible abolition of circadian HRV rhythm, } \\
\text { loss of relative vagal nocturnal dominance. }\end{array}$ \\
\hline Giubilei et al., 1998 [62] & $\begin{array}{l}10 \text { patients with MCA infarct vs. controls, } 1 \text { day after } \\
\text { stroke, power spectrum analysis of HRV during sleep. }\end{array}$ & $\begin{array}{l}\text { Sympathetic/parasympathetic balance (VLF+HF/HF), } \\
\text { higher in patients. Sympathetic predominance during } \\
\text { sleep. }\end{array}$ \\
\hline Korpelainen et al., 1999 [60] & $\begin{array}{l}46 \text { stroke patients (early: } 1-7 \text { days poststroke; follow up at } \\
6 \text { month) vs. } 30 \text { controls. } 24 \text { h Holter. }\end{array}$ & $\begin{array}{l}\text { Abnormal HRV measures involved in cardiac complica- } \\
\text { tions. SDNN, VLF, LF impaired in acute and late state } \\
\text { post stroke. }\end{array}$ \\
\hline Tokgözoglu et al., 1999 [58] & $\begin{array}{l}62 \text { stroke patients vs. } 62 \text { matched controls. } 72 \mathrm{~h} \text { ECG re- } \\
\text { cording post stroke. Power spectrum analysis. }\end{array}$ & $\begin{array}{l}\text { Decreased LF, HF, SDNN. Decreased sympathetic and } \\
\text { parasympathetic parameters, most pronounced in right } \\
\text { insular cortex infarcts. }\end{array}$ \\
\hline Colivicchi et al., 2004 [55] & $\begin{array}{l}103 \text { stroke patients, } 24 \mathrm{~h} \text { Holter, time and frequency do- } \\
\text { main HRV. }\end{array}$ & $\begin{array}{l}\text { Lower SDNN, rMSSD; higher LF/HF ratio in right } \\
\text { insular infarct patients in association with more frequent } \\
\text { and complex arrhythmias. }\end{array}$ \\
\hline Mäkikallio et al., 2004 [63] & $\begin{array}{l}84 \text { stroke patients, } 24 \mathrm{~h} \text { Holter, follow up } 7 \text { years, power- } \\
\text { law slope beta (beta<-1.5). }\end{array}$ & $\begin{array}{l}\text { Conventional HRV measures without prognostic power, } \\
\text { but abnormal long-term HR dynamics predict poststroke } \\
\text { mortality. }\end{array}$ \\
\hline Meyer et al., 2004 [64] & 29 stroke patients within $24 \mathrm{~h}$ after event, HR and BP. & $\begin{array}{l}\text { Pathological sympathetic activation more pronounced } \\
\text { after right sided stroke. }\end{array}$ \\
\hline Colivicchi et al., 2005 [57] & 208 stroke patients, $24 \mathrm{~h}$ Holter, 12 month follow up. & $\begin{array}{l}\text { Decreased SDNN, right insular infarct and non sustained } \\
\text { ventricular tachycardia associated with higher risk of } \\
\text { early mortality. }\end{array}$ \\
\hline McLaren et al., 2005 [56] & $\begin{array}{l}\text { Cross sectional case-control study in } 76 \text { stroke patients ( } 9 \\
\text { months poststroke) vs. } 70 \text { matched controls; long term } \\
\text { effects; power spectrum analysis, baro reflex sensitivity, } \\
\text { cardiovascular reflex autonomic test. }\end{array}$ & $\begin{array}{l}\text { Impaired autonomic function may increase mortality in } \\
\text { older stroke survivors. }\end{array}$ \\
\hline Bassi et al., 2007 [65] & 85 stroke patients, $24 \mathrm{~h}$ Holter, time domain measure. & $\begin{array}{l}\text { Lower SDNN associated with unfavourable outcome } \\
\text { after rehabilitation. }\end{array}$ \\
\hline Dütsch et al., 2007 [66] & $\begin{array}{l}28 \text { stroke patients } v s .21 \text { matched controls, RR intervals, } \\
\text { BP means oscillations. }\end{array}$ & $\begin{array}{l}\text { Post stroke parasympathetic cardiac deficit and increased } \\
\text { sympathetic cardiovascular modulation. LF/HF ratio of } \\
\text { RR intervals elevated in right hemispheric vs. left sided } \\
\text { stroke. }\end{array}$ \\
\hline Rufa et al., 2007 [67] & $\begin{array}{l}23 \text { CADASIL patients vs. } 22 \text { controls, frequency domain } \\
\text { measures of HRV, QTc Interval. }\end{array}$ & $\begin{array}{l}\text { LF/HF ratio higher in CADASIL patients. No correlation } \\
\text { with MRI lesion volume. }\end{array}$ \\
\hline
\end{tabular}


creased HRV and increased heart rate) [55]. This ANS dysfunction was associated with higher incidence of sudden death [58].

The prognostic importance of a sympathetic activation post stroke was demonstrated in more than 100 patients studied by [59]. An elevated norepinephrine concentration was associated with insular involvement, nocturnal elevated blood pressure and prolonged QTc interval in the ECG. One year post stroke these patients showed a higher incidence of cardio- and cerebrovascular events, a lower degree of neurological restitution of stroke symptoms in conjunction with lower rate of independence [59].

Relevant studies analyzing stroke associated changes in ANS activity and of those assessing the prognostic value of HRV indices after stroke based on 24 hours ECG recordings are reviewed in Table 3 .

Predictors for a lethal outcome are diminished heart rate variability (HRV), insular cortex involvement, and the occurrence of complex arrhythmias within $72 \mathrm{~h}$ after admission [55,57]. A decreased nocturnal vagal dominance leading to hypertension and decreased HRV during night-time resulted in a long lasting autonomic dysfunction and thus chronic risk [60]. The standard deviation of all normal to normal R-R (NN) intervals (SDNN) is a very strong prognostic predictor, together with the cardiac arrhythmia rate [55,57,60,61].

In summary, a number of experimental and clinical studies have revealed that ANS dysfunction represents a relevant pathogenetic factor and indicator for post stroke morbidity and mortality. Thus, using HRV related indices in acute and postacute stroke victims may improve prognostic evaluation and also counseling of patients, and may thus eventually lead to new preventive strategies recognizing the important relationship of ANS dysfunction and central nervous system injury.

\section{CONCLUSIONS}

Heart rate patterns which can easily be obtained from Holter ECG recordings reflect important characteristics of the complex autonomic control. They are not only associated with cardiac arrhythmias, but implicate sympathetic and parasympathetic modulations.

In stroke and cardiovascular disease as well as in other very relevant clinical constellations, such as in sepsis and MODS (multiple organ dysfunction syndrome; e.g. [14,25] a selective pathological disturbance leads to a complex systemic compensation and adaptation process. Consequently, final decompensation and respective risk assessment might be related rather to an overall systemic response than to a particular mechanism, unless this is fatally altered itself. Therefore, it is not surprising, that for heart failure / MI patients the comprehensive HRV measures like SDNN, VLF, beta slope, complexity, and AIF measures were found to have the best predictive value (as reviewed above). On the other hand, specific indices, such as the shifted adjustment between sympathetic and vagal activity (sympatho-vagal (im)-balance) due to stroke and cardiovascular diseases provided predictive value with more specific physiological implications.

From the clinical point of view the main question is, whether HRV indices provide additional pathophysiological and predictive value going beyond the conventional risk factors and clinical parameters which are so far not completely satisfying in particular in severely and acutely ill patients. This objective becomes even more challenging since multiple "cardiovascular" confounders, such as diabetes, age, daytime, as well as pharmacological interventions like betablockers, angiotensin-converting enzyme (ACE) inhibitors, and statins which modulate autonomic tone and systemic proinflammatory responses [68] have to be considered.

Besides the autonomic modulations, which are assessed following the established HRV guidelines [6], cardiac arrhythmias such as atrial fibrillation, sinoatrial dysfunction and ectopic complexes are of essential prognostic value and their patterns are subject of current research. Especially after stroke the HRV-index SDNN and the presence of nonsustained ventricular tachycardia were independent predictors of the 1-year mortality [57]. Cardiogenic arrhythmias and autonomic modulations cannot be completely separated, because of the complex autonomic-cardiovascular system, but both aspects have to be considered in a way appropriate for the respective situation, such as sinus rhythm or cardiac arrhythmia. Furthermore, stroke related immunodepression [2] has to be considered as another important player in this relationship between ANS, CNS and cardiovascular system.

Due to the multiple influences on the autonomic function and the rapid development of confounding or targeted therapies large scale clinical studies are immediately necessary to further evaluate the clinical value of the above mentioned HRV indices in cardio- and cerebrovascular risk assessment und management.

\section{ACKNOWLEDGEMENT}

We thank Dr. Hischam Taha, Frenchay Hospital, Bristol, for helpful comments.

\section{REFERENCES}

[1] Stein PK. Potential role of different components of heart rate variability for risk-stratification in critical care. Crit Care Med 2005; 33(9): 2128-30.

[2] Meisel C, Schwab JM, Prass K, Meisel A, Dirnagl U. Central nervous system injury-induced immune deficiency syndrome. Nat Rev Neurosci 2005; 6(10): 775-86.

[3] Borovikova LV, Ivanova S, Zhang M, et al. Vagus nerve stimulation attenuates the systemic inflammatory response to endotoxin. Nature 2000; 405(6785): 458-62.

[4] Hasko G, Szabo C. Regulation of cytokine and chemokine production by transmitters and co-transmitters of the autonomic nervous system. Biochem Pharmacol 1998 ; 56(9): 1079-87.

[5] Woiciechowsky C, Asadullah K, Nestler D, et al. Sympathetic activation triggers systemic interleukin-10 release in immunodepression induced by brain injury. Nat Med 1998; 4(7): 808-13.

[6] TaskForce. Heart rate variability: standards of measurement, physiological interpretation and clinical use. Task Force of the European Society of Cardiology and the North American Society of Pacing and Electrophysiology. Circulation 1996 ; 93(5): $1043-$ 65.

[7] Akselrod S, Cerutti S, Honzikova N, et al. Analyses of cardiovascular oscillations for enhanced diagnosis and risk stratification in cardiac diseases and disorders. Biomed Tech (Berl) 2006; 51(4): 276-8.

[8] Bigger JT, Jr, Steinman RC, Rolnitzky LM, Fleiss JL, Albrecht P, Cohen RJ. Power law behavior of RR-interval variability in healthy middle-aged persons, patients with recent acute myocardial infarction, and patients with heart transplants. Circulation 1996; 93(12): 2142-51. 
[9] Peng CK, Havlin S, Stanley HE, Goldberger AL. Quantification of scaling exponents and crossover phenomena in nonstationary heartbeat time series. Chaos1995; 5(1): 82-7.

[10] Pincus SM. Approximate entropy as a measure of system complexity. Proc Natl Acad Sci USA 1991; 88(6): 2297-301.

[11] Richman JS, Moorman JR. Physiological time-series analysis using approximate entropy and sample entropy. Am J Physiol Heart Circ Physiol $2000 ; 278(6)$ : H2039-49.

[12] Porta A, Baselli G, Liberati D, et al. Measuring regularity by means of a corrected conditional entropy in sympathetic outflow. Biol Cybern 1998; 78(1): 71-8.

[13] Hoyer D, Bauer R, Pompe B, et al. Nonlinear Analysis of the Cardiorespiratory coordination in a newborn piglet. In: Kantz $\mathrm{H}$, Kurths J, Mayer-Kress G, Eds. Nonlinear analysis of physiological data. Berlin: Springer; 1998. pp. 167-90.

[14] Hoyer D, Friedrich H, Zwiener U, et al. Prognostic impact of autonomic information flow in multiple organ dysfunction syndrome patients. Int J Cardiol 2006 14; 108(3): 359-69.

[15] Hoyer D, Pompe B, Chon KH, Hardraht H, Wicher C, Zwiener U. Mutual information function assesses autonomic information flow of heart rate dynamics at different time scales. IEEE Trans Biomed Eng 2005 ; 52(4): 584-92.

[16] Kurths J, Voss A, Saparin P, Witt A, Kleiner HJ, Wessel N. Quantitative analysis of heart rate variability. Chaos $1995 ; 5(1)$ : 88-94.

[17] Voss A, Kurths J, Kleiner HJ, et al. The application of methods of non-linear dynamics for the improved and predictive recognition of patients threatened by sudden cardiac death. Cardiovasc Res 1996; 31(3): 419-33.

[18] Lake DE, Richman JS, Griffin MP, Moorman JR. Sample entropy analysis of neonatal heart rate variability. Am J Physiol Regul Integr Comp Physiol 2002 ; 283(3): R789-97.

[19] Costa M, Goldberger AL, Peng CK. Multiscale entropy analysis of complex physiologic time series. Phys Rev Lett 2002; 89(6): 068102.

[20] Hoyer D, Friedrich H, Frank B, et al. Autonomic information flow improves prognostic impact of task force HRV monitoring. Comput Methods Progr Biomed 2006 ; 81(3): 246-55.

[21] Hoyer D, Friedrich H, Stein PK, et al. Autonomic information flow improves prognostic value of heart rate patterns after abdominal aortic surgery. J Crit Care 2008 ; 23(2): 255-62.

[22] Schmidt G, Malik M, Barthel P, et al. Heart-rate turbulence after ventricular premature beats as a predictor of mortality after acute myocardial infarction. Lancet 1999; 353(9162): 1390-6.

[23] Barthel P, Schneider R, Bauer A, et al. Risk stratification after acute myocardial infarction by heart rate turbulence. Circulation 2003; 108(10): 1221-6.

[24] Kleiger RE, Stein PK, Bigger JT, Jr. Heart rate variability: measurement and clinical utility. Ann Noninvasive Electrocardiol 2005; 10(1): $88-101$

[25] Schmidt H, Muller-Werdan U, Hoffmann T, et al. Autonomic dysfunction predicts mortality in patients with multiple organ dysfunction syndrome of different age groups. Crit Care Med 2005 ; 33(9): 1994-2002.

[26] La Rovere MT, Pinna GD, Raczak G. Baroreflex sensitivity: measurement and clinical implications. Ann Noninvasive Electrocardiol 2008; 13(2): 191-207.

[27] Pagani M, Somers V, Furlan R, et al. Changes in autonomic regulation induced by physical training in mild hypertension. Hypertension 1988; 12(6): 600-10.

[28] Parati G, Di Rienzo M, Bertinieri G, et al. Evaluation of the baroreceptor-heart rate reflex by 24 -hour intra-arterial blood pressure monitoring in humans. Hypertension 1988; 12(2): 214-22.

[29] Taylor JA, Carr DL, Myers CW, Eckberg DL. Mechanisms underlying very-low-frequency RR-interval oscillations in humans. Circulation 1998 ; 98(6): 547-55.

[30] Bigger JT, Jr, Fleiss JL, Steinman RC, Rolnitzky LM, Kleiger RE, Rottman JN. Correlations among time and frequency domain measures of heart period variability two weeks after acute myocardial infarction. Am J Cardiol 1992; 69(9): 891-8.

[31] Piccirillo G, Ogawa M, Song J, et al. Power spectral analysis of heart rate variability and autonomic nervous system activity measured directly in healthy dogs and dogs with tachycardia-induced heart failure. Heart Rhythm 2009; 6(4): 546-52.

[32] Stein PK. Measures of parasympathetic function and risk stratification in critical care. Crit Care Med 2008; 36(3): 1025-7.
[33] La Rovere MT, Bigger JT, Jr, Marcus FI, Mortara A, Schwartz PJ. Baroreflex sensitivity and heart-rate variability in prediction of total cardiac mortality after myocardial infarction. ATRAMI (Autonomic Tone and Reflexes After Myocardial Infarction) Investigators. Lancet 1998; 351(9101): 478-84.

[34] Carney RM, Blumenthal JA, Freedland KE, et al. Low heart rate variability and the effect of depression on post-myocardial infarction mortality. Arch Intern Med 2005; 165(13): 1486-91.

[35] Penzel T, Kantelhardt JW, Lo CC, Voigt K, Vogelmeier C. Dynamics of heart rate and sleep stages in normals and patients with sleep apnea. Neuropsychopharmacology 2003; Suppl 1: S48-53.

[36] Rajendra AU, Paul JK, Kannathal N, Lim CM, Suri JS. Heart rate variability: a review. Med Biol Eng Comput 2006; 44(12): 103151 .

[37] Burr RL. Interpretation of normalized spectral heart rate variability indices in sleep research: a critical review. Sleep 2007; 30(7): $913-$ 9.

[38] Aubert AE, Seps B, Beckers F. Heart rate variability in athletes. Sports Med 2003; 33(12): 889-919.

[39] Hoyer D, Frank B, Baranowski R, Zebrowski JJ, Stein PK, Schmidt H. Autonomic information flow rhythms. From heart beat interval to circadian variation. IEEE Eng Med Biol Mag 2007; 26(6): 1924.

[40] Ivanov P. Scale-invariant aspects of cardiac dynamics: observing sleep stages and circadian phases. IEEE Eng Med Biol Mag 2007; 26(6): 33-7.

[41] Lemmer B. Circadian rhythm regulations of the cardiovascular system. Studies in rats and mice. IEEE Eng Med Biol Mag 2007; 26(6): 30-2.

[42] Stein PK, Lundequam EJ, Oliveira LP, et al. Cardiac autonomic modulation. Analyzing circadian and ultradian rhythms. IEEE Eng Med Biol Mag 2007; 26(6): 14-8.

[43] Makikallio TH, Hoiber S, Kober L, et al. Fractal analysis of heart rate dynamics as a predictor of mortality in patients with depressed left ventricular function after acute myocardial infarction. TRACE Investigators. TRAndolapril Cardiac Evaluation. Am J Cardiol 1999; 83(6): 836-9.

[44] Maestri R, Pinna GD, Porta A, et al. Assessing nonlinear properties of heart rate variability from short-term recordings: are these measurements reliable? Physiol Meas 2007 28(9): 1067-77.

[45] Hoyer D, Maestri R, La Rovere MT, Pinna GD. Autonomic response to cardiac dysfunction in chronic heart failure - a risk predictor based on autonomic information flow. Pacing Clin Electrophysiol 2008; 31(2): 214-20.

[46] Goldberger JJ, Cain ME, Hohnloser SH, et al. American Heart Association/American College of Cardiology Foundation/Heart Rhythm Society scientific statement on noninvasive risk stratification techniques for identifying patients at risk for sudden cardiac death: a scientific statement from the American Heart Association Council on Clinical Cardiology Committee on Electrocardiography and Arrhythmias and Council on Epidemiology and Prevention. Circulation 2008; 118(14): 1497-518.

[47] Nolan J, Batin PD, Andrews R, et al. Prospective study of heart rate variability and mortality in chronic heart failure: results of the United Kingdom heart failure evaluation and assessment of risk trial (UK-heart). Circulation 1998; 98(15): 1510-6.

[48] Galinier M, Pathak A, Fourcade J, et al. Depressed low frequency power of heart rate variability as an independent predictor of sudden death in chronic heart failure. Eur Heart J 2000; 21(6): 475-82.

[49] La Rovere MT, Pinna GD, Maestri R, et al. Short-term heart rate variability strongly predicts sudden cardiac death in chronic heart failure patients. Circulation 2003; 107(4): 565-70.

[50] Guzzetti S, La Rovere MT, Pinna GD, et al. Different spectral components of $24 \mathrm{~h}$ heart rate variability are related to different modes of death in chronic heart failure. Eur Heart J 2005; 26(4): 357-62.

[51] Lanza GA, Cianflone D, Rebuzzi AG, et al. Prognostic value of ventricular arrhythmias and heart rate variability in patients with unstable angina. Heart 2006; 92(8): 1055-63.

[52] Johnston KC, Li JY, Lyden PD, et al. Medical and neurological complications of ischemic stroke: experience from the RANTTAS trial. RANTTAS Investigators. Stroke 1998 ; 29(2): 447-53.

[53] Barron SA, Rogovski Z, Hemli J. Autonomic consequences of cerebral hemisphere infarction. Stroke 1994 25(1): 113-6.

[54] Naver HK, Blomstrand C, Wallin BG. Reduced heart rate variability after right-sided stroke. Stroke. 1996; 27(2): 247-51. 
[55] Colivicchi F, Bassi A, Santini M, Caltagirone C. Cardiac autonomic derangement and arrhythmias in right-sided stroke with insular involvement. Stroke 2004; 35(9): 2094-8.

[56] McLaren A, Kerr S, Allan L, et al. Autonomic function is impaired in elderly stroke survivors. Stroke 2005; 36(5): 1026-30.

[57] Colivicchi F, Bassi A, Santini M, Caltagirone C. Prognostic implications of right-sided insular damage, cardiac autonomic derangement, and arrhythmias after acute ischemic stroke. Stroke 2005; 36(8): 1710-5

[58] Tokgozoglu SL, Batur MK, Top uoglu MA, Saribas O, Kes S, Oto A. Effects of stroke localization on cardiac autonomic balance and sudden death. Stroke 1999; 30(7): 1307-11.

[59] Sander D, Winbeck K, Klingelhofer J, Etgen T, Conrad B. Prognostic relevance of pathological sympathetic activation after acute thromboembolic stroke. Neurology 2001; 57(5): 833-8.

[60] Korpelainen JT, Sotaniemi KA, Makikallio A, Huikuri HV, Myllyla VV. Dynamic behavior of heart rate in ischemic stroke. Stroke 1999; 30(5): 1008-13

[61] Korpelainen JT, Sotaniemi KA, Huikuri HV, Myllyla VV. Circadian rhythm of heart rate variability is reversibly abolished in ischemic stroke. Stroke 1997; 28(11): 2150-4.
[62] Giubilei F, Strano S, Lino S, et al. Autonomic nervous activity during sleep in middle cerebral artery infarction. Cerebrovasc Dis 1998; 8(2): 118-23.

[63] Makikallio AM, Makikallio TH, Korpelainen JT, Sotaniemi KA, Huikuri HV, Myllyla VV. Heart rate dynamics predict poststroke mortality. Neurology 2004; 62(10): 1822-6.

[64] Meyer S, Strittmatter M, Fischer C, Georg T, Schmitz B. Lateralization in autonomic dysfunction in ischemic stroke involving the insular cortex. Neuroreport 2004; 15(2): 357-61.

[65] Bassi A, Colivicchi F, Santini M, Caltagirone C. Cardiac autonomic dysfunction and functional outcome after ischaemic stroke. Eur J Neurol 2007; 14(8): 917-22.

[66] Dutsch M, Burger M, Dorfler C, Schwab S, Hilz MJ. Cardiovascular autonomic function in poststroke patients. Neurology 2007; 69(24): 2249-55

[67] Rufa A, Guideri F, Acampa M, et al. Cardiac autonomic nervous system and risk of arrhythmias in cerebral autosomal dominant arteriopathy with subcortical infarcts and leukoencephalopathy (CADASIL). Stroke 2007; 38(2): 276-80.

[68] Schmidt H, Hoyer D, Wilhelm J, et al. The alteration of autonomic function in multiple organ dysfunction syndrome. Crit Care Clin $2008 ; 24(1): 149-63$.

Received: May 11, 2009

Revised: December 22, 2009

Accepted: February 04, 2010

(c) Günther et al.; Licensee Bentham Open.

This is an open access article licensed under the terms of the Creative Commons Attribution Non-Commercial License (http://creativecommons.org/licenses/by-nc/3.0/) which permits unrestricted, non-commercial use, distribution and reproduction in any medium, provided the work is properly cited. 